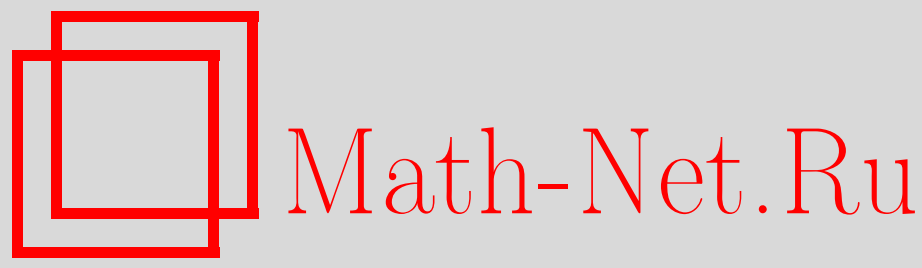

Ф. Галли, А. С. Кошелев, Многополевая космологическая модель в струнной теории поля: точно решаемое приближение, ТМФ, 2010, том 164, номер 3, 401-409

DOI: https://doi.org/10.4213/tmf6550

Использование Общероссийского математического портала Math-Net.Ru подразумевает, что вы прочитали и согласны с пользовательским соглашением http://www . mathnet.ru/rus/agreement

Параметры загрузки:

IP: 52.90 .164 .192

26 апреля 2023 г., 16:58:42

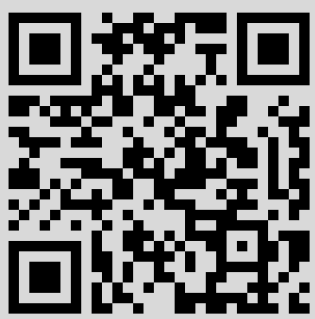




\title{
ФИЗИКА
}

Том 164, № 3

сентябрь, 2010

2010 г.

Ф. Галли*, А. С. Кошелев*

\section{МНОГОПОЛЕВАЯ КОСМОЛОГИЧЕСКАЯ МОДЕЛЬ В СТРУННОЙ ТЕОРИИ ПОЛЯ: ТОЧНО РЕШАЕМОЕ ПРИБЛИЖКНИЕ}

\begin{abstract}
Рассматривается появление многих скалярных полей в основанных на струнной теории поля нелокальных моделях с одним скалярным полем при больших временах. В этом режиме все скалярные поля являются свободными. Такая система, минимально связанная с гравитацией, может быть проанализирована приближенно или численно. Построена точно решаемая модель, которая имеет точное решение в космологическом сценарии с метрикой Фридмана и воспроизводит асимптотическое поведение, ожидаемое из струнной теории поля. Рассматриваются различные приложения такого потенциала в многополевых космологических моделях.
\end{abstract}

Ключевые слова: струнная теория поля, космологические возмущения, фантом.

\section{1. ВВЕДЕНИЕ}

В настоящей статье мы рассматриваем новый класс космологических моделей, основанных на струнной теории поля (СТП) (см., например, обзоры [1]) и $p$-адической теории струн [2]. Мы строим точно решаемую модель, воспроизводящую поведение исходной модели в асимптотическом режиме. Более детальный анализ можно найти в статьях [3]-[6] (см. также приведенную там литературу). Известно, что СТП и $p$-адическая теория струн являются УФ-конечными. Тем самым можно ожидать, что получающиеся из них (эффективные) модели являются свободными от патологий. Более того, модели, основанные на СТП, обладают общим нестандартным свойством, а именно: они содержат члены с бесконечным числом производных, т.е. нелокальные члены. Высшие производные обычно порождают хорошо известные нестабильности Остроградского [7]. Однако результат Остроградского относится к производным выше второго, но конечного порядка. В случае бесконечного числа производных возможно, что нестабильности не возникают.

Имеющиеся данные космологических наблюдений [8], [9] явно подтверждают, что современная Вселенная испытывает ускоренное расширение, тем самым подтверждается существование темной энергии [10]. Последние результаты эксперимента

*Vrije Universiteit Brussel; The International Solvay Institutes, Brussels, Belgium.

E-mail: fgalli@tena4.vub.ac.be, alexey.koshelev@vub.ac.be 
WMAP [9] вместе с данными по сверхновым типа Іа ставят следующие ограничения на параметр состояния темной энергии: $w_{\mathrm{DE}}=-1.02_{-0.16}^{+0.14}$. Отметим, что существующие эксперименты не исключают, что параметр $w$ изменяется со временем. Нелокальные модели, получаемые из СТП, могут иметь эффективное фантомное поведение и тем самым представляют интерес для современной космологии. Для построения стабильной модели с $w<-1$ требуется построить эффективную теорию с нарушением условия нулевой энергии исходя из фундаментальной теории, которая стабильна и допускает квантование. Это аргумент в пользу космологических моделей, получаемых из СТП.

\section{2. МОДЕЛЬ}

Мы будем исследовать нелокальные модели, возникающие в СТП в режиме больших времен. Отправной точкой является лагранжиан вида

$$
S=\int d^{4} x \sqrt{-g}\left(\frac{R}{16 \pi G_{N}}+\frac{1}{g_{o}^{2}}\left(-\frac{1}{2} \partial_{\mu} T \partial^{\mu} T+\frac{1}{2 \alpha^{\prime}} T^{2}-\frac{1}{\alpha^{\prime}} v(\bar{T})\right)-\Lambda^{\prime}\right),
$$

где явно указана размерность пространства-времени $4, G_{N}$ - ньютоновская константа, $8 \pi G_{N}=1 / M_{\mathrm{P}}^{2}$, где $M_{\mathrm{P}}$ - масса Планка, $\alpha^{\prime}$ - длина струны в квадрате (мы не отождествляем $\left.\alpha^{\prime}=1 / M_{\mathrm{P}}^{2}\right), g_{o}$ - константа связи открытых струн, $g_{\mu \nu}-$ метрический тензор, $R$ - скалярная кривизна, $\Lambda^{\prime}-$ постоянный член, $\bar{T}=\mathcal{G}\left(\alpha^{\prime} \square\right) T$ с $\square=D^{\mu} \partial_{\mu}=\frac{1}{\sqrt{-g}} \partial_{\mu} \sqrt{-g} g^{\mu \nu} \partial_{\nu}, D_{\mu}-$ ковариантная производная, $T-$ скалярное поле, которое наиболее естественно отождествляется с тахионом открытой струны, $v(\bar{T})$ - самодействие тахиона открытой струны, не содержащее квадратичного по полю $T$ члена. Мы работаем в пространстве размерности $1+3$ с сигнатурой $(-,+,+,+)$, координаты имеют греческие индексы $\mu, \nu, \ldots$, изменяющиеся в диапазоне от 0 до 3. Пространственные индексы обозначаются $a, b, \ldots$ и изменяются от 1 до 3. Такое четырехмерное действие мотивировано СТП (подробнее см. [4]). Функция $\mathcal{G}\left(\alpha^{\prime} \square\right)$ может быть неполиномиальной, порождая тем самым явную нелокальность. В СТП $\mathcal{G}\left(\alpha^{\prime} \square\right)=e^{-\beta \alpha^{\prime} \square / 2}$, где $\beta$ - параметр, определяемый исключительно конформной теорией поля струны, но мы не будем привязываться к конкретному виду этой функции. Очень важная особенность, которую мы здесь видим, - нелокальность в действии. Существенно, что появление нелокальности является общим свойством моделей, основанных на СТП. Именно это свойство лежит в основе рассматриваемых в этой статье моделей ${ }^{1)}$. Поля являются безразмерными, в то время как размерность величины $g_{o}$ есть длина. Фактор $1 / \alpha^{\prime}$ перед потенциалом тахиона выглядит необычно, но от него можно легко избавиться с помощью масштабного преобразования полей. Для нас удобнее иметь безразмерные поля.

Вводя поле $T_{b}=\bar{T}$ и обезразмеренные координаты $\bar{x}_{\mu}=x_{\mu} / \sqrt{\alpha^{\prime}}$, можно переписать введенное выше действие следующим образом (мы опускаем черту над координатами и полагаем в дальнейшем $\left.\alpha^{\prime}=1\right)$ :

$$
S=\int d^{4} x \sqrt{-g}\left(\frac{R}{16 \pi G_{N}}+\frac{1}{g_{o}^{2}}\left(-\frac{1}{2} \partial_{\mu} \widetilde{T}_{b} \partial^{\mu} \widetilde{T}_{b}+\frac{1}{2} \widetilde{T}_{b}^{2}-v\left(T_{b}\right)\right)-\Lambda^{\prime}\right) .
$$

\footnotetext{
1) Появление высших производных не является исключительно свойством СТП. Некоммутативные теории поля, например, также содержат высшие производные, но соответствующие нелокальные структуры совершенно другие.
} 
Подчеркнем, что потенциал поля $T_{b}$ - это $V=-T_{b}^{2} / 2+v\left(T_{b}\right)$. Предполагая наличие экстремума потенциала $V$, можно линеаризовать теорию в его окрестности, используя равенство $T_{b}=T_{0}-\tau$. В результате получим

$$
V=-\frac{1}{2} \tau^{2}+\frac{v\left(T_{0}\right)^{\prime \prime}}{2} \tau^{2}+V\left(T_{0}\right)
$$

Действие (2), линеаризованное в окрестности экстремума потенциала поля $T_{b}$, может быть записано в виде

$$
S=\int d^{4} x \sqrt{-g}\left(\frac{R}{16 \pi G_{N}}+\frac{1}{2 g_{o}^{2}} \tau \mathcal{F}(\square) \tau-\Lambda\right),
$$

где $\mathcal{F}(\square)=(\square+1) \mathcal{G}^{-2}(\square)-m^{2}, m^{2} \equiv v\left(T_{0}\right)^{\prime \prime}$ и $\Lambda=\Lambda^{\prime}+V\left(T_{0}\right) / g_{o}^{2}$. Из СТП имеем $\mathcal{F}_{\mathrm{SFT}}(\square)=(\square+1) e^{\beta \square}-m^{2}$. Функция $\mathcal{F}$, в сущности, является обратным пропагатором, и естественно ожидать, что $\beta<0$ в случае СТП, это соответствует сходящемуся пропагатору при больших импульсах. На этом примере из СТП следует очень важный вывод. Пусть $m=0$. Тогда $\mathcal{F}_{\mathrm{SFT}}=(\square+1) e^{\beta \square}$, и нелокальность никак не проявляется. В самом деле, экспонента не изменяет полюсную структуру пропагатора. Другими словами, это означает, что экспоненциальный фактор можно убрать переопределением полей. Ситуация кардинально отличается в случае $m \neq 0$. Пропагатор $1 / \mathcal{F}_{\mathrm{SFT}}$ имеет бесконечно много полей. Это и есть проявление нелокальности. Также очевидно, что физические картины будут абсолютно различными в случае полной функции $\mathcal{F}$ и при представлении ее в виде ряда с последующим обрезанием, так как структура полюсов может существенно измениться.

Мы видим, что важную роль играет спектр теории, определяемый уравнением

$$
\mathcal{F}(J)=(J+1) \mathcal{G}^{-2}(J)-m^{2}=0 .
$$

Мы будем называть его характеристическим уравнением. Оно может быть алгебраическим или трансцендентным. Мы рассматриваем $\mathcal{F}(\square)$ в общем виде. Единственное предположение, которое мы делаем, это то, что все корни являются простыми. Также для нас важна аналитичность функции $\mathcal{F}$ на комплексной плоскости, т. е. возможность представить $\mathcal{F}$ в виде сходящегося ряда вида

$$
\mathcal{F}=\sum_{n=0}^{\infty} f_{n} \square^{n}, \quad f_{n} \in \mathbb{R} .
$$

Требование вещественности коэффициентов следует из требования эрмитовости лагранжиана. Строго говоря, даже требование аналитичности может быть ослаблено, но в этом случае нужно аккуратно рассматривать полюсы и соответствующие области сходимости ряда.

Классические решения уравнений движения были изучены в работе [4]. Существенным моментом в этом анализе является тот факт, что действие (3) полностью эквивалентно следующему действию со многими локальными свободными скалярными полями:

$$
S_{\text {local }}=\int d^{4} x \sqrt{-g}\left(\frac{R}{16 \pi G_{N}}-\frac{1}{g_{o}^{2}} \sum_{i} \frac{\mathcal{F}^{\prime}\left(J_{i}\right)}{2}\left(g^{\mu \nu} \partial_{\mu} \tau_{i} \partial_{\nu} \tau_{i}+J_{i} \tau_{i}^{2}\right)-\Lambda\right) .
$$


Здесь $J_{i}$ - корни характеристического уравнения (4), всего полей столько, сколько корней имеет характеристическое уравнение. Подробный анализ вопроса эквивалентности может быть найден в работах [4], [6], [11].

Важно отметить, что как корни $J_{i}$, так и коэффициенты $\mathcal{F}^{\prime}\left(J_{i}\right)$ могут быть комплексными. Это не является проблемой, так как все локальные поля - всего лишь математические функции, не имеющие физической интерпретации. Важно, чтобы исходное поле $\tau$ являлось вещественным, так как оно представляет собой физическое возбуждение. Этого легко добиться. Действительно, корни $J_{i}$ либо вещественные, либо образуют комплексно-сопряженные пары. Комплексно-сопряженные корни $J_{i}$ дадут комплексно-сопряженные (с точностью до общего множителя) решения для $\tau_{i}$. Тем самым линейная комбинация полей $\tau_{i}$ может быть сделана вещественной путем подбора констант интегрирования. Кроме того, коэффициенты $\mathcal{F}^{\prime}\left(J_{i}\right)$ можно убрать с помощью масштабного преобразования полей.

Далее мы будем рассматривать локальное действие (6). Более того, мы ограничиваем наше рассмотрение пространственно-плоской Вселенной Фридмана-Робертсона-Уокера. Метрика имеет вид

$$
d s^{2}=-d t^{2}+a^{2}(t)\left(d x_{1}^{2}+d x_{2}^{2}+d x_{3}^{2}\right)
$$

где $a(t)$ - масштабный фактор, $t$ - космическое время. Параметр Хаббла определяется как обычно: $H=\dot{a} / a$, точка здесь и далее означает производную по космическому времени $t$. Фоновые решения для $\tau$ берутся пространственно однородными.

В работе [6] было доказано, что космологические возмущения в свободной теории с одним нелокальным скалярным полем (3) и в соответствующей локальной теории (6) со многими скалярными полями также являются эквивалентными. Более того, было изучено несколько примеров эволюции системы и соответствующих возмущений. Трудность в том, что известно мало точно решаемых моделей со многими скалярными полями. Однако изучать возмущения, не имея точного решения, сложно. Конечно, не обязательно иметь бесконечно много полей. Напротив, есть возможность положить почти все поля равными нулю, выбрав нулевые константы интегрирования. Проблема в том, что модели, в которых точное решение известно, это в основном модели с одним полем. Сведение действия (6) к модели с одним полем полностью скрывает богатую нетривиальную структуру, пришедшую из СТП. Наиболее интересный случай комплексных масс требует по крайней мере двух полей. В самом деле, необходимо, чтобы исходная функция $\tau$ была вещественной, поэтому кроме одного комплексного поля нужен еще его комплексно-сопряженный партнер.

\section{3. ТОЧНО РЕШАЕМАЯ МОДЕЛЬ}

Точное аналитическое решение уравнений движения, следующих из действия (6) (и тем более из (2)), неизвестно. Однако в случае, когда ставится задача изучения космологических возмущений, гораздо легче работать с точными решениями, а не с асимптотическим поведением. Есть шанс, тем не менее, изменить потенциал так, что, во-первых, модель станет точно решаемой и, во-вторых, в интересующем нас 
режиме новые члены будут пренебрежимо малы, и тем самым восстановится исходная модель. Более того, точно решаемая модель имеет самостоятельную ценность просто потому, что не так много точно решаемых моделей известно в космологии. В нашем случае мы имеем дело со многими полями, и это еще более усложняет задачу. Также мы имеем дело с комплексными коэффициентами в лагранжиане, что является неизученной проблемой.

Рассмотрим действие

$$
\begin{aligned}
S= & \int d^{4} x \sqrt{-g}\left[\frac{R}{16 \pi G_{N}}+\frac{1}{g_{o}^{2}}\left(-\sum_{i} \frac{\mathcal{F}^{\prime}\left(J_{i}\right)}{2}\left(g^{\mu \nu}\left(\partial_{\mu} \tau_{i+} \partial_{\nu} \tau_{i+}+\partial_{\mu} \tau_{i-} \partial_{\nu} \tau_{i-}\right)+\right.\right.\right. \\
& \left.\left.\left.+J_{i}\left(\tau_{i+}^{2}+\tau_{i-}^{2}\right)\right)-\frac{3 \pi G}{2}\left(\sum_{i} \mathcal{F}^{\prime}\left(J_{i}\right)\left(\alpha_{i+} \tau_{i+}^{2}+\alpha_{i-} \tau_{i-}^{2}\right)\right)^{2}+\text { c.c. }\right)-\Lambda\right],
\end{aligned}
$$

где $H_{0}=\sqrt{8 \pi G_{N} \Lambda / 3}, G \equiv G_{N} / g_{o}^{2}-$ безразмерный аналог ньютоновской константы, $\alpha_{i \pm}$ - два решения ${ }^{2)}$ уравнения $J_{i}=-\alpha_{i}\left(\alpha_{i}+3 H_{0}\right)$.

Для упрощения обозначений введем индексы $P, Q, R, \ldots$, учитывающие вырождение в $\alpha_{i}$, т. е. будем считать $P, Q, R, \ldots=i_{+}, i_{-}, j_{+}, \ldots$, имея в виду, что теперь возможно равенство $J_{P}=J_{Q}$ для некоторых значений $P$ и $Q$. С такими обозначениями действие (8) имеет вид

$$
\begin{aligned}
S= & \int d^{4} x \sqrt{-g}\left(\frac{R}{16 \pi G_{N}}+\frac{1}{g_{o}^{2}}\left(-\widetilde{\sum_{P}} \frac{\mathcal{F}^{\prime}\left(J_{P}\right)}{2}\left(g^{\mu \nu} \partial_{\mu} \tau_{P} \partial_{\nu} \tau_{P}+J_{P} \tau_{P}^{2}\right)-\right.\right. \\
& \left.\left.-\frac{3 \pi G}{2}\left(\widetilde{\sum_{P}} \mathcal{F}^{\prime}\left(J_{P}\right) \alpha_{P} \tau_{P}^{2}\right)^{2}\right)-\Lambda\right),
\end{aligned}
$$

где $\widetilde{\sum}_{P}$ означает, что комплексно-сопряженные величины включены в суммирование $^{3)}$.

Локальное действие имеет точное решение соответствующих ему уравнений движения, которые имеют вид

$$
\begin{aligned}
3 H^{2} & =4 \pi G\left(\widetilde{\sum_{P}} \mathcal{F}^{\prime}\left(J_{P}\right)\left(\dot{\tau}_{P}^{2}+J_{P} \tau_{P}^{2}\right)+3 \pi G\left(\widetilde{\sum_{P}} \mathcal{F}^{\prime}\left(J_{P}\right) \alpha_{P} \tau_{P}^{2}\right)^{2}\right)+8 \pi G_{N} \Lambda, \\
\dot{H} & =-4 \pi G \widetilde{\sum_{P}} \mathcal{F}^{\prime}\left(J_{P}\right) \dot{\tau}_{P}^{2},
\end{aligned}
$$

и

$$
\ddot{\tau}_{P}+3 H \dot{\tau}_{P}+J_{P} \tau_{P}+6 \pi G \alpha_{P} \tau_{P} \widetilde{\sum_{Q}} \mathcal{F}^{\prime}\left(J_{Q}\right) \alpha_{Q} \tau_{Q}^{2}=0 \quad \text { для всех } P \text {. }
$$

2) В частном случае $4 J^{2} /\left(9 H_{0}^{2}\right)=1$ два решения совпадают и практически остается лишь одно скалярное поле.

${ }^{3)}$ Например, $\widetilde{\Sigma}_{P} J_{P} \tau_{P}^{2}=\sum_{P}\left(J_{P} \tau_{P}^{2}+J_{P}^{*} \tau_{P}^{2 *}\right)$. 
Можно проверить явно, что имеется следующее решение ${ }^{4)}$ :

$$
\begin{aligned}
\tau_{P} & =\tau_{P 0} e^{\alpha_{P} t}, \\
H & =H_{0}-2 \pi G \widetilde{\sum_{P}} \mathcal{F}^{\prime}\left(J_{P}\right) \alpha_{P} \tau_{P 0}^{2} e^{2 \alpha_{P} t} .
\end{aligned}
$$

Это решение справедливо для любого числа полей (включая одно поле) и для любых значений параметров $\alpha_{P}$ (т. е. вещественных или комплексных). Также видно, что в случае $\operatorname{Re} \alpha_{P}<0$ для всех $P$ член четвертого порядка в потенциале скалярных полей исчезает и остаются только свободные поля. Тем самым при больших временах модель (6) восстанавливается и можно говорить об асимптотическом режиме моделей, основанных на СТП. Стабильность полученных решений заслуживает более глубокого анализа, для этого могут быть применены методы, используемые в работе [13].

\section{4. КОСМОЛОГИЧЕСКИЕ ВОЗМУЩЕНИЯ}

Построим уравнения, описывающие космологические возмущения для точного решения (12). Для действия (9) получаются следующие уравнения для возмущений в случае многих скалярных полей:

$$
\begin{aligned}
& \ddot{\zeta}_{P Q}+\dot{\zeta}_{P Q}\left(3 H+\frac{\ddot{\tau}_{P}}{\dot{\tau}_{P}}+\frac{\ddot{\tau}_{Q}}{\dot{\tau}_{Q}}\right)+\zeta_{P Q}\left(-3 \dot{H}+\frac{k^{2}}{a^{2}}\right)= \\
& =\left[\frac{\tau_{P}}{\dot{\tau}_{P}}\left(J_{P}+6 \pi G \alpha_{P} \widetilde{\sum_{R}} \mathcal{F}^{\prime}\left(J_{R}\right) \alpha_{R} \tau_{R}^{2}\right)-\right.
\end{aligned}
$$

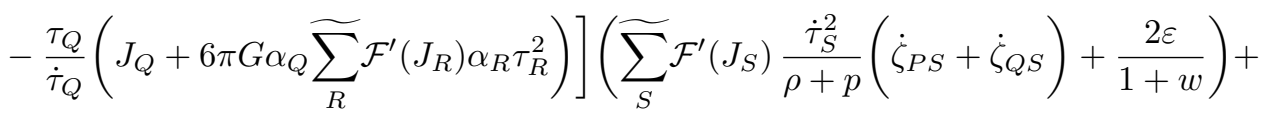

$$
\begin{aligned}
& +12 \pi G \widetilde{\sum_{R}} \mathcal{F}^{\prime}\left(J_{R}\right) \alpha_{R} \tau_{R} \dot{\tau}_{R}\left(\alpha_{P} \frac{\tau_{P}}{\dot{\tau}_{P}} \zeta_{P R}-\alpha_{Q} \frac{\tau_{Q}}{\dot{\tau}_{Q}} \zeta_{Q R}\right), \\
& \ddot{\varepsilon}+\dot{\varepsilon} H\left(2-6 w+3 c_{\mathrm{s}}^{2}\right)+\varepsilon\left(\dot{H}(1-3 w)-15 w H^{2}+9 H^{2} c_{\mathrm{s}}^{2}+\frac{k^{2}}{a^{2}}\right)= \\
& =\frac{k^{2}}{a^{2}} \frac{1}{\rho} \frac{2}{\rho+p} \widetilde{\sum_{R, S}}\left(J_{R}+6 \pi G \alpha_{R} \widetilde{\sum_{P}} \mathcal{F}^{\prime}\left(J_{P}\right) \alpha_{P} \tau_{P}^{2}\right) \mathcal{F}^{\prime}\left(J_{R}\right) \mathcal{F}^{\prime}\left(J_{S}\right) \tau_{R} \dot{\tau}_{R} \dot{\tau}_{S}^{2} \zeta_{R S} .
\end{aligned}
$$

Здесь $\zeta_{P Q}=\delta \tau_{P} / \dot{\tau}_{P}-\delta \tau_{Q} / \dot{\tau}_{Q}-$ калибровочно-инвариантная переменная, описывающая возмущение скалярных полей, $\varepsilon$ - калибровочно-инвариантное возмущение полной плотности энергии, $k$ - волновое число (в работах [14]-[16], [6] можно найти вывод уравнений для возмущений). Также мы используем следующие обозначения: $w \equiv p / \rho-$ параметр уравнения состояния, $c_{\mathrm{s}}^{2} \equiv \dot{p} / \dot{\rho}-$ скорость звука. Общие плотности энергии и давления имеют вид

$$
\rho=\frac{1}{2} \widetilde{\sum_{P}} \mathcal{F}^{\prime}\left(J_{P}\right) \dot{\tau}_{P}^{2}+V, \quad p=\frac{1}{2} \widetilde{\sum_{P}} \mathcal{F}^{\prime}\left(J_{P}\right) \dot{\tau}_{P}^{2}-V,
$$

\footnotetext{
4) Похожая модификация действия была рассмотрена в работе [12], где был предпринят анализ в случае вещественных корней.
} 
где

$$
V=\frac{1}{2} \widetilde{\sum_{P}} \mathcal{F}^{\prime}\left(J_{P}\right) J_{P} \tau_{P}^{2}+\frac{3 \pi G}{2}\left(\widetilde{\sum_{P}} \mathcal{F}^{\prime}\left(J_{P}\right) \alpha_{P} \tau_{P}^{2}\right)^{2} .
$$

Используя точное решение (12), находим

$$
\begin{aligned}
& \rho=\frac{1}{2} \widetilde{\sum_{P}} \mathcal{F}^{\prime}\left(J_{P}\right) \tau_{0 P}^{2} e^{2 \alpha_{P} t}\left(\alpha_{P}^{2}+J_{P}\right)+\frac{3 \pi G}{2}\left(\widetilde{\sum_{P}} \mathcal{F}^{\prime}\left(J_{P}\right) \alpha_{P} \tau_{0 P}^{2} e^{2 \alpha_{P i} t}\right)^{2}+g_{0}^{2} \Lambda= \\
& =\frac{3}{8 \pi G} H^{2} \text {, } \\
& p=\frac{1}{2} \widetilde{\sum_{P}} \mathcal{F}^{\prime}\left(J_{P}\right) \tau_{0 P}^{2} e^{2 \alpha_{P} t}\left(\alpha_{P}^{2}-J_{P}\right)-\frac{3 \pi G}{2}\left(\widetilde{\sum_{P}} \mathcal{F}^{\prime}\left(J_{P}\right) \alpha_{P} \tau_{0 P}^{2} e^{2 \alpha_{P} t}\right)^{2}-g_{0}^{2} \Lambda= \\
& =-\frac{\dot{H}}{4 \pi G}-\frac{3}{8 \pi G} H^{2},
\end{aligned}
$$

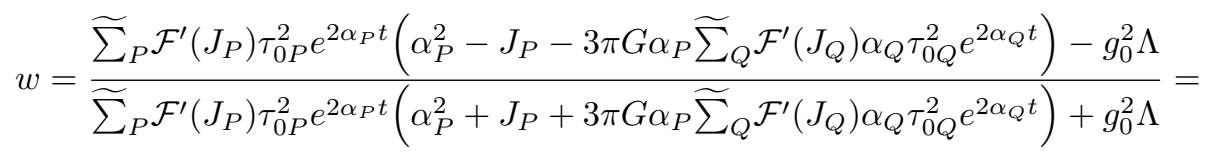

$$
\begin{aligned}
& =-1-\frac{2}{3} \frac{\dot{H}}{H^{2}}, \\
& c_{\mathrm{s}}^{2}=\frac{{\widetilde{\sum_{P}}}_{P} \mathcal{F}^{\prime}\left(J_{P}\right) \alpha_{P} \tau_{0 P}^{2} e^{2 \alpha_{P} t}\left(\alpha_{P}^{2}-J_{P}-6 \pi G \widetilde{\sum_{Q}} \mathcal{F}^{\prime}\left(J_{Q}\right) \alpha_{Q}^{2} \tau_{0 Q}^{2} e^{2 \alpha_{Q} t}\right)}{\widetilde{\sum}_{P} \mathcal{F}^{\prime}\left(J_{P}\right) \alpha_{P} \tau_{0 P}^{2} e^{2 \alpha_{P} t}\left(\alpha_{P}^{2}+J_{P}+6 \pi G \widetilde{\sum_{Q}} \mathcal{F}^{\prime}\left(J_{Q}\right) \alpha_{Q}^{2} \tau_{0 Q}^{2} e^{2 \alpha_{Q} t}\right)}=-1-\frac{\ddot{H}}{3 \dot{H} H} .
\end{aligned}
$$

Подставляя точное решение для $\tau_{P}$ в уравнение (13), видим, что член, соответствующий второй производной потенциала, в правой части сокращается с членом, пропорциональным $\dot{H}$, в левой части. Уравнение (14), вычисленное на точном решении (12), упрощается, так как в этом случае вклад от члена четвертого порядка в потенциале пропадает. Уравнения можно переписать, используя функцию $H$ и ее производные, а также выразить все $J_{P}$ как $-\alpha_{P}\left(\alpha_{P}-3 H_{0}\right)$. Члены с $H_{0}$ пропадают по соображениям симметрии выражения, содержащего $J_{P}$ :

$$
\begin{aligned}
\ddot{\zeta}_{P Q} & +\dot{\zeta}_{P Q}\left(3 H+\alpha_{P}+\alpha_{Q}\right)+\zeta_{P Q} \frac{k^{2}}{a^{2}}= \\
& =\frac{1}{\dot{H}}\left(\alpha_{P}-\alpha_{Q}\right)\left(4 \pi G \widetilde{\sum_{R}} \mathcal{F}^{\prime}\left(J_{R}\right) \tau_{0 R}^{2} \alpha_{R}^{2} e^{2 \alpha_{R} t}\left(\dot{\zeta}_{P R}+\dot{\zeta}_{Q R}\right)+3 H^{2} \varepsilon\right), \\
\ddot{\varepsilon}+\dot{\varepsilon} & \left(5 H+4 \frac{\dot{H}}{H}-\frac{\ddot{H}}{\dot{H}}\right)+\varepsilon\left(6 H^{2}+14 \dot{H}+2 \frac{\dot{H}^{2}}{H^{2}}-3 H \frac{\ddot{H}}{\dot{H}}+\frac{k^{2}}{a^{2}}\right)= \\
& =\frac{k^{2}}{a^{2}} \frac{4}{3} \frac{(4 \pi G)^{2}}{\sum_{R, S}} \mathcal{F}^{\prime}\left(J_{R}\right) \mathcal{F}^{\prime}\left(J_{S}\right) \alpha_{R} \alpha_{R}^{2} \alpha_{S}^{2} \tau_{0 R}^{2} \tau_{0 S}^{2} e^{2 \alpha_{R} t} e^{2 \alpha_{S} t} \zeta_{R S} .
\end{aligned}
$$

Последние два уравнения представляют основной результат настоящей статьи. Анализ этих уравнений является важной задачей, находящейся в процессе решения.

Один пример возмущений с комплексными корнями в исходном линеаризованном действии (6) был рассмотрен в работе [17]. Линейные возмущения в такой конфигурации мы можем ограничить, не разрушая тем самым систему. Такой результат не 
очевиден и согласуется с утверждением, что модели, основанные на СТП, являются стабильными. Случай комплексных $J_{P}$ никогда не изучался и заслуживает более глубокого исследования. Анализ возмущений с комплексными корнями в точно решаемой модели будет предпринят в последующей работе.

Благодарности. А. С. Кошелев благодарит организаторов конференции "Problems of Theoretical and Mathematical Physics" за возможность представить настоящую работу и за создание благоприятной обстановки для научных дискуссий. Авторы благодарны И.Я. Арефьевой, Ф.Л. Безрукову, Б. Крапсу, Б. Драговичу, Г. Двали, В.Ф. Муханову и С. Ю. Вернову за ценные замечания и плодотворное обсуждение. Работа частично поддержана The Belgian Federal Science Policy Office через программу The Interuniversity Attraction Poles IAP VI/11, The European Commission FP6 RTN через программу MRTN-CT-2004-005104 и FWO-Vlaanderen (проект G.0428.06).А.С.Кошелев частично поддержан РФФИ (грант № 08-01-00798) и Российским федеральным агентством по науке и инновациям (государственный контракт 02.740.11.5057). Ф. Галли является аспирантом FWO-Vlaanderen, a A. C. Koшелев - исследователем FWO-Vlaanderen.

\section{Список литературы}

[1] K. Ohmori, A review on tachyon condensation in open string field theories, arXiv: hep-th/0102085; I. Ya. Aref'eva, D. M. Belov, A. A. Giryavets, A. S. Koshelev, P. B. Medvedev, Noncommutative field theories and (super) string field theories, arXiv: hep-th/0111208; W. Taylor, Lectures on D-branes, tachyon condensation, and string field theory, arXiv: hep-th/0301094.

[2] L. Brekke, P. G. O. Freund, M. Olson, E. Witten, Nucl. Phys. B, 302:3 (1988), 365-402; P. H. Frampton, Ya. Okada, Phys. Rev. D, 37:10 (1988), 3077-3079; В. С. Владимиров, И. В. Волович, Е. И. Зеленов, р-Адический анализ и математическая физика, Наука, M., 1994; B. Dragovich, A. Yu. Khrennikov, S. V. Kozyrev, I. V. Volovich, Ultrametric Anal. Appl., 1:1 (2009), 1-17, arXiv: 0904.4205.

[3] I. Ya. Aref'eva, "Nonlocal string tachyon as a model for cosmological dark energy", p-Adic Mathematical Physcis, AIP Conf. Proc., 826, eds. A. Yu. Khrennikov, Z. Rakić, I. V. Volovich, AIP, New York, 2006, 301-311, arXiv: astro-ph/0410443; I. Ya. Aref'eva, "Stringy model of cosmological dark energy", Particles, Strings, and Cosmology, AIP Conf. Proc., 957, eds. A. Rajantie, P. Dauncey, C. Contaldi, H. Stoica, AIP, New York, 2007, 297-300, arXiv: 0710.3017.

[4] A. S. Koshelev, JHEP, 04 (2007), 029, 19 pp., arXiv: hep-th/0701103.

[5] I. Ya. Aref'eva, A.S. Koshelev, JHEP, 09 (2008), 068, 20 pp., arXiv: 0804.3570.

[6] A. S. Koshelev, S. Yu. Vernov, Cosmological perturbations in SFT inspired non-local scalar field models, arXiv: 0903.5176.

[7] M. Ostrogradski, Mem. Ac. St. Petersburg, VI, 4 (1850), 385.

[8] S. J. Perlmutter, G. Aldering, G. Goldhaber et al., Astrophys. J., 517:2 (1999), 565-586, arXiv: astro-ph/9812133; A. G. Riess, A. V. Filippenko, P. Challis et al., Astron. J., 116:3 (1998), 1009-1038, arXiv: astro-ph/9805201; A. G. Riess, L.-G. Strolger, J. Tonry et al., Astrophys. J., 607:2 (2004), 665-687, arXiv: astro-ph/0402512; R. A. Knop, G. Aldering, R. Amanullah, et al., Astrophys. J., 598:1 (2003), 102-137, arXiv: astro-ph/0309368; M. Tegmark et al. (the SDSS collaboration), Phys. Rev. D, 69:10 (2004), 103501, 26 pp., arXiv: astro-ph/0310723v2; D. N. Spergel, L. Verde, H. V. Peiris et al., Astrophys. J. Suppl., 148:1 (2003), 175-194, arXiv: astro-ph/0302209; P. Astier, J. Guy, 
N. Regnault et al., Astron. Astrophys., 447:1 (2006), 31-48, arXiv: astro-ph/0510447; W. M. Wood-Vasey, G. Miknaitis, C. W. Stubbs et al., Astrophys. J., 666:2 (2007), 694-715, arXiv: astro-ph/0701041.

[9] E. Komatsu, J. Dunkley, M. R. Nolta et al., Astrophys. J. Suppl., 180:2 (2009), 330-376, arXiv: 0803.0547; M. Kilbinger, K. Benabed, J. Guy et al., Astron. Astrophys., 497:3 (2009), 677-688, arXiv: 0810.5129.

[10] T. Padmanabhan, Phys. Rep., 380:5-6 (2003), 235-320, arXiv: hep-th/0212290; P.H. Frampton, Dark energy - a Pedagogic Review, arXiv: astro-ph/0409166; E. J. Copeland, M. Sami, Sh. Tsujikawa, Internat. J. Modern Phys., 15:11 (2006), 1753-1935, arXiv: hep-th/0603057; A. Albrecht, G. Bernstein, R. Cahn et al., Report of the dark energy task force, arXiv: astro-ph/0609591.

[11] I. Ya. Aref'eva, L. V. Joukovskaya, S. Yu. Vernov, J. Phys. A, 41:30 (2008), 304003, 14 pp., arXiv: 0711.1364; D. J. Mulryne, N. J. Nunes, Phys. Rev. D, 78:6 (2008), 063519, 16 pp., arXiv: 0805.0449.

[12] I. Ya. Aref'eva, L. V. Joukovskaya, S. Yu. Vernov, JHEP, 07 (2007), 087, 36 pp., arXiv: hep-th/0701184.

[13] I. Ya. Aref'eva, N. V. Bulatov, S. Yu. Vernov, Stable exact solutions in cosmological models with two scalar fields, arXiv: 0911.5105.

[14] J. M. Bardeen, Phys. Rev. D, 22:8 (1980), 1882-1905.

[15] V.F. Mukhanov, H. A. Feldman, R. H. Brandenberger, Phys. Rep., 215:5-6 (1992), 203-333; V.F. Mukhanov, Physical Foundations of Cosmology, Cambridge Univ. Press, Cambridge, 2005.

[16] J. Hwang, H. Noh, Class. Quant. Grav., 19:3 (2002), 527-549, arXiv: astro-ph/0103244.

[17] A.S. Koshelev, SFT non-locality in cosmology: solutions, perturbations and observational evidences, arXiv: 0912.5457. 